\title{
Teriparatide improves outcomes of periodontal surgery
}

A short course of teriparatide, given in conjunction with periodontal surgery, could help rebuild bone and improve clinical outcomes in patients with severe, chronic periodontitis, say researchers from the University of Michigan.

The research group, led by

Laurie McCauley, a Professor in the Department of Periodontics and Oral Medicine, had previously shown in animal models that bone formed during wound healing is particularly responsive to the anabolic actions of parathyroid hormone. "Armed with this information, we identified patients with inflammatory bone disease (periodontitis) requiring surgical intervention as the ideal target population in which to translate our laboratory findings to a clinical reality," McCauley explains. The aim of the current study was to assess the ability of teriparatide-a drug made up of the first 34 amino acids of parathyroid hormone-to promote osseous regeneration in the oral cavity.

The investigators enrolled 40 patients with periodontitis in their study, which employed a double-blind, randomized, placebo-controlled design. Participants received either teriparatide or placebo once-daily for a 6-week period, starting 3 days before surgery, plus supplementation with calcium and vitamin $\mathrm{D}$. The surgical procedure comprised a standard open-flap debridement, where gingival tissues are reflected and the bone defects and tooth roots thoroughly cleaned. The patients were followed for 1 year; outcome measures included radiographic linear measurement of alveolar bone level and periodontal probing depth (a measure of the health of the tissues surrounding the teeth).

\section{4 ...even a short dosing of} this drug had benefits that lasted a year... 77

McCauley's team made three important observations during the follow-up period. First, treatment with teriparatide led to greater resolution of ossesous defects (29\% gain in bone height versus $3 \%$ with placebo). Second, teriparatide improved clinical attachment levels around the tooth. Finally, use of teriparatide was associated with greater reductions in periodontal probing depth than was observed in the group that received placebo.
Remarkably, the reported effects of teriparatide persisted and/or further improved throughout follow-up.

"I think one really interesting aspect of this study is that even a short dosing of this drug had benefits that lasted a year," comments McCauley.

The investigators now plan to assess the effect of teriparatide on craniofacial bone at the histological level in patients undergoing placement of dental implants. They are also evaluating local delivery strategies in preclinical models, using scaffold materials to provide targeted approaches to treatment.

The findings of the current trial suggest that teriparatide could help promote healing in the oral cavity. "This study supports a rationale for further investigations, such as the use of teriparatide for situations where patients with compromised wound healing (for example, osteonecrosis of the jaw) may benefit," McCauley concludes.

Vicky Heath

Original article Bashutski, J. D. et al. Teriparatide and osseous regeneration in the oral cavity. N. Engl. J. Med. doi:10.1056/NEJMoa1005361 